\title{
A DIGITAL EDUCATIONAL GAME FOR PRACTICING OER EDITING
}

\author{
Lubna Ali ${ }^{1,2}$, Vu Tuan $\operatorname{Tran}^{2}$, Rene Roepke $e^{1,2}$ and Ulrik Schroeder ${ }^{1,2}$ \\ ${ }^{1}$ Learning Technologies Research Group \\ ${ }^{2} R$ WTH Aachen University \\ Aachen, Germany
}

\begin{abstract}
The concept of Open Educational Resources (OER) was introduced to the world more than twenty years ago. Despite the fact that countless efforts have been made to promote the development of OER since then, the awareness of OER around the world is still considerably low. Many educators, who are familiar with the concept of OER, still hesitate to use OER in teaching and educational practices because they are concerned of violating copyright laws. This is mainly due to the lack of knowledge and practice in working with the OER. To disseminate the knowledge of OER, we have applied Game-based learning (GBL) and implemented a digital educational game that does not only help players practice editing and combining different OERs using suitable licenses but also motivates them to discover the world of OER by themselves. The game will be integrated within the introductory OER Workshops held at RWTH Aachen University. In this paper, we present the educational game and illustrate its main functionalities.
\end{abstract}

\section{KEYWORDS}

Open Educational Resources, Game-based Learning, Creative Commons, Educational Game, Licensing

\section{INTRODUCTION}

According to the United Nations Educational, Scientific, and Cultural Organization (UNESCO), the right to education is an international human right. Universal education is considered as one of the most important targets to be achieved besides providing people with food, clean water, and basic accommodation (UNESCO 2021). It is not only one of the most powerful tools for eradicating poverty but also the primary key to sustainable and peaceful development in the world. Nevertheless, according to the most recent reports on the website of UNESCO, there are still more than 262 million children and young people, who cannot go to school (UNESCO 2021). While their parents struggle to provide them with food and basic accommodation, they also cannot afford expensive textbooks and education. To help break out the cycle of poverty and lack of education, educational resources must be freely available to everyone. Based on this idea, the concept of Open Educational Resources (OER) was born.

OER are free learning materials that can be accessed by anyone (UNESCO 2021). They are presented in many forms such as documents, research papers, videos, audio files, images, slides, etc. Some of the most well-known platforms for distributing OER are YouTube, Wikimedia Commons, and MIT Open Course Ware. OER do not only benefit the users by delivering high-quality learning content without any cost but also the content creators (Caswell 2008). Although OER can be accessed free of charge, creators can still set the terms of use for their content by providing the OER with suitable licenses. In most cases, OER are licensed with Creative Commons (CC) licenses. CC licenses were introduced and are maintained by the non-profit organization Creative Commons, which helps people overcome legal difficulties in producing and using OER. Due to the human readability of the licenses, users can easily figure out what they are allowed to do and not.

In practice, people often mix different OER to create their own resources. Here, we consider two types of OER combinations: the composition and the collage. In the composition, each used OER has a clear and complete reference to its original source so that users can easily distinguish the different OERs used within the composition. Contradictory, collage is the kind of content, in which the resources are mixed and users can hardly determine the original resources. 
While it is rather easy to combine OER if they have the same CC licenses, combining OER licensed under different licenses can be quite challenging. To support people in practicing the editing of OER, we have deployed a Game-based Learning (GBL) concept to develop an educational game that does not only convey the knowledge of OER but also motivates them to discover the world of OER themselves. In this paper, we will introduce the game and illustrate how it can be used within introductory OER workshops to support the participants in practicing and understanding the concept of OER Editing.

\section{RELATED WORK}

There are various tools that can be used when combining or editing different OERs. One of these tools is the Creative Commons Mixer (CC Mixer ${ }^{1}$ ), which is an online web service used for checking if a combination of different $\mathrm{CC}$ licenses is valid. Because the CC Mixer is just a fast-developed product for a hackathon, the usage of the CC Mixer is still very limited. For example, the CC Mixer assumes that all combinations are collages. Another tool that also supports users in properly licensing their creative work is the Creative Commons Chooser $\left(\mathrm{CC} \mathrm{Chooser}{ }^{2}\right)$. CC Chooser is also an online tool that helps people choose the right CC license for their work. In general, CC Chooser has a friendlier User Interface (UI) than CC Mixer. However, CC Chooser was built for people who create the whole content on their own without using any available OER and want to publish their work with a CC license. This is quite dangerous because there is no clear hint on the website of CC Chooser reminding the user to pay attention to the licenses of external OER used.

Overall, existing tools that support the creation and editing of OER are either limited in functionality or do not provide support in combining existing OER using the correct licensing. Therefore, these tools are not suitable to present to people learning about OER and starting to create and edit OER. In addition, they fail to motivate and encourage people to explore the world of OER. In our attempt to provide a new tool supporting users to create and edit OER, we decided to apply Game-based Learning (GBL), as it helps users interact with learning environment and motivate people to explore and practice presented learning content. Indeed, the efficiency of GBL was verified by many experiments conducted in real life.

In (Rahmah 2019), Dr. Rahmah Fithriani at the Universitas Islam Negeri Sumatera Utara turned her grammar class into a playground, in which students learn English through various types of game namely Guessing the Word, Board \& Dice Game, and Run \& Guess. After the course, a survey was conducted to verify the efficiency of the game-based teaching method. The result was very positive. Before the course, more than 93\% of the students said that they did not like learning grammar. After the course, all of them changed their opinion and said that they loved learning grammar. Before the course, less than $24 \%$ of students thought that grammar was necessary for communication. However, after the course, more than $96 \%$ of them agree that grammar was very helpful. Besides the aforementioned experiment, Mas-Machuca, M. et al. applied GBL in a Human Resources Management (HRM) course during the academic year of 2018/2019 at Universitat Internacional de Catalunya, Spain (Mas-Machuca 2019). Instead of purely teaching academic theory, they turned the course into a competition, in which students have to be active outside the classroom in order to win. After the course, a survey was conducted to evaluate the efficiency of the new approach. Results show that all the students prefer the game-based method to the traditional one. The authors claim that GBL not only helps them understand how the theory can be applied in real life but also strengthens the bond among students.

\section{PROTOTYPE}

One does not simply give the learners a game containing educational content and then expect increased motivation or significantly improved learning results. For that reason, we chose to follow the guidelines in (Plass 2015) to design a game that fits to the target audiences and the learning content. For simplicity, we chose to build a question-based game. The game consists of various multiple-choice questions about OER and CC license system.

\footnotetext{
http://ccmixer.edu-sharing.org/, last accessed on 02.08.2021

https://creativecommons.org/choose/?lang=de, last accessed on 02.08.2021
} 
Regarding the narrative, we created a storyline that takes place in medieval time to distract players from the fact that they are learning about OER and immerse them into a thematic environment. In the story, as the best smiths in the realm, players are asked to forge a sword for the King. We choose this storyline because there are many similarities in the process of creating/editing OER to the process of forging a sword. For example, the process of mixing different material to create steel is very similar to the process of mixing different OER to create new OER.

Furthermore, to motivate the players to achieve the best in-game result, we built a leaderboard that honors the best players with the highest scores. The score of each level is calculated based on the performance of the player as well as the time they spend on the level. Regarding the learning content, the game is structured from easy to difficult.

In the first three levels, players are supported with practices. These practices are not only for preparing the players before jumping into the main storyline but also for reviewing the knowledge they learned in the OER workshops. In the main levels, players will have to apply their understanding of editing OER to overcome the challenge.

The target group of our game comes from various fields and is interested in editing or creating their own OER. We assume that most players already have basic knowledge about OER and the CC license system but do not have much practical experience with OER. Figure 1 shows a screenshot of the game interface.

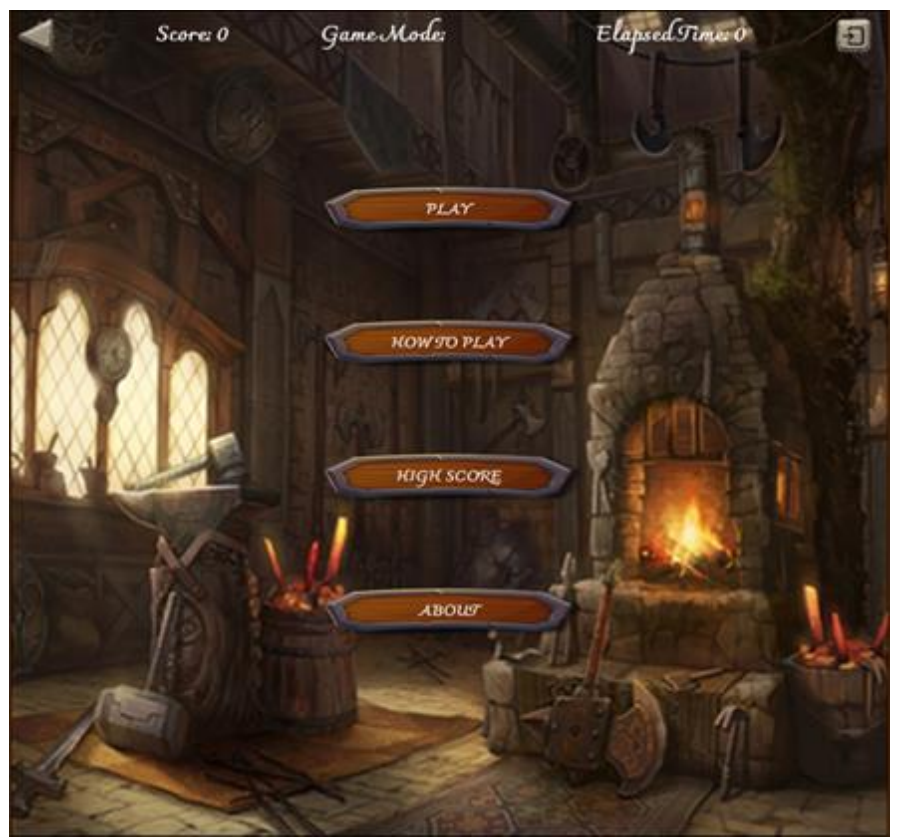

Figure 1. Screenshot of the game interface

Our game was developed as web-based game that can run in most modern browsers. The architecture behind the game consists of three main components: The frontend, the backend, and the database. The frontend is used to control and present the user interface. The backend serves as a bridge connecting the frontend and the database. The database is used to store the leaderboard information and user data.

\section{USE CASE FOR EVALUATION}

As a first testbed for our game, we consider the participants of an introductory workshop about OER organized by the Learning Technologies Research Group at RWTH Aachen University. The participants are either academic educators at the university or students in teacher education programs, who will become teachers in the schools after they graduate. The game will be introduced within the workshops as a tool to help the participants practice their knowledge about OER they have acquired in the workshop. The game is therefore utilized as a practical assignment. The workshop organizers will provide access and sufficient time to play the 
game (10 to 15 minutes). After playing the game, we plan to evaluate it by using a short survey. Players can then evaluate the functionality and user experience of the game. Open-ended questions allow for qualitative feedback regarding improvement ideas for the game. This way, we can explore how to extend our game for future workshops.

\section{CONCLUSION AND FUTURE WORK}

One of the barriers hindering the development of OER is the lack of knowledge about OER and, consequently, lack of practice in using OER. GBL is a concept that proposes the use of games in education. Through many studies, GBL has proven its advantages over traditional teaching methods. Besides conveying knowledge to the learners, GBL can increase their engagement in the learning process and encourage them to seek knowledge themselves.

Realizing the potential of GBL we have developed a digital educational game that supports players in practicing OER editing interactively during introductory OER workshops. The game was carefully designed, so that even people who do not have much experience with editing OER and the CC licensing system should be able to play and learn from the game without any problems. The game's user interface was implemented to simplify the interaction between the players and the game as much as possible, so that the users do not need to read the instruction to know how to play the game. To motivate the players to strive for the best result, we added a leaderboard honoring the best players as an incentive system to the game. For further investigation and evaluation, we will gather feedback from the participants in upcoming OER workshops

Although the game has fulfilled our initial requirements of helping people practice OER editing, there is still plenty of room for extensions. To make the game more attractive, the storyline could be expanded or even a second one could be added. Another storyline would not only increase the complexity of the game, but also help players to understand OER better through providing more practice opportunities. Regarding the used game mechanics, our current game prototype is based on simple question mechanic. The interactions between the players and the game are very simple. The advantage of this simplicity is that the users do not need to read the instruction to know how to play. However, because of its simplicity, the players might get easily bored and lose interest. One suggestion for the improvement is to add more methods for players to interact with the game such as controllable avatars and different game mechanics.

Conclusively, we have developed a game that not only help players practice editing OER but also motivate them to discover the world of OER and CC licensing. Currently, we are planning to extend the game so that it can help people practice not only editing OER, but also the thematic fields of searching, using, and creating OER. Through this game, we have indirectly contributed to the promotion of the development of OER and advocated the right to education for everyone.

\section{REFERENCES}

Boyle, E. et al, 2016. An update to the systematic literature review of empirical evidence of the impacts and outcomes of computer games and serious games, Computers \& Education 94, 178-192.

Caswell, T. et al, 2008. Open Educational Resources: Enabling universal education, International Review of Research in Open and Distance Learning Vol 9 No. 1.

Mas-Machuca, M. et al, 2019. Implementation of Game-based Learning in Higher Education: an example in HR Management, 5th International Conference on Higher Education Advances (HEAd'19), pp. 1043-1050.

Plass, J. et al. Foundations of Game-Based Learning, Educational Psychologist: Vol 50, No. 4, 258-283 (2015).

Rahmah, F, 2019. Communicative game-based learning in EFL grammar class: Suggested activities and students' perception, Journal of English Education and Linguistics Studies 5(2), 171-188.

UNESCO (2021). Right to education. Available at: https://en.unesco.org/themes/right-to-education, (Accessed: 30 June 2021).

UNESCO (2021). Open Educational Resources (OER). Available at: https://en.unesco.org/themes/building-knowledgesocieties/oer, (Accessed: 29 June 2021). 